\title{
Point-by-Point model description of average prompt neutron data as a function of total kinetic energy of fission fragments
}

\author{
A. Tudora \\ University of Bucharest, Faculty of Physics, Bucharest-Magurele, RO-77125, Romania
}

\begin{abstract}
The experimental data of average prompt neutron multiplicity as a function of total kinetic energy of fragments $\left\langle v>\right.$ (TKE) exhibit, especially in the case of ${ }^{252} \mathrm{Cf}(\mathrm{SF})$, different slopes $\mathrm{dTKE} / \mathrm{d} v$ and different behaviours at low TKE values. The Point-by-Point ( $\mathrm{PbP})$ model can describe these different behaviours. The higher slope dTKE/d $v$ and the flattening of $\langle v\rangle$ at low TKE exhibited by a part of experimental data sets is very well reproduced when the $\mathrm{PbP}$ multi-parametric matrix $v(\mathrm{~A}, \mathrm{TKE})$ is averaged over a double distribution $\mathrm{Y}(\mathrm{A}, \mathrm{TKE})$. The lower slope and the almost linear behaviour over the entire TKE range exhibited by other data sets is well described when the same matrix $v(\mathrm{~A}, \mathrm{TKE})$ is averaged over a single distribution $\mathrm{Y}(\mathrm{A})$. In the case of average prompt neutron energy in SCM as a function of TKE, different $\mathrm{dTKE} / \mathrm{d} \varepsilon$ slopes are also obtained by averaging the same $\mathrm{PbP}$ matrix $\varepsilon(\mathrm{A}, \mathrm{TKE})$ over $\mathrm{Y}(\mathrm{A}, \mathrm{TKE})$ and over $\mathrm{Y}(\mathrm{A})$. The results are exemplified for three fissioning systems benefiting of experimental data as a function of TKE: ${ }^{252} \mathrm{Cf}(\mathrm{SF}),{ }^{235} \mathrm{U}\left(\mathrm{n}_{\mathrm{th}}, \mathrm{f}\right)$ and ${ }^{239} \mathrm{Pu}\left(\mathrm{n}_{\mathrm{th}}, \mathrm{f}\right)$. In the case of ${ }^{234} \mathrm{U}(\mathrm{n}, \mathrm{f})$ for the first time it was possible to calculate $\left\langle v>\right.$ (TKE) and $\left\langle_{\varepsilon}>\right.$ (TKE) at many incident energies by averaging the $\mathrm{PbP}$ multi-parametric matrices over the experimental $\mathrm{Y}(\mathrm{A}, \mathrm{TKE})$ distributions recently measured at IRMM for 14 incident energies in the range 0.3 $5 \mathrm{MeV}$. The results revealed that the slope dTKE/d $v$ does not vary with the incident energy and the flattening of $\langle v\rangle$ at low TKE values is more pronounced at low incident energies. The average model parameters dependences on TKE resulted from the PbP treatment allow the use of the most probable fragmentation approach, having the great advantage to provide results at many TKE values in a very short computing time compared to $\mathrm{PbP}$ and Monte Carlo treatments.
\end{abstract}

\section{Point-by-Point calculation of average prompt neutron data as a function of total kinetic energy of fission fragments}

The existing experimental data as a function of TKE of ${ }^{252} \mathrm{Cf}(\mathrm{SF})$ and ${ }^{235} \mathrm{U}\left(\mathrm{n}_{\mathrm{th}}, \mathrm{f}\right)$ exhibit different behaviours. The experimental $<\nu>$ (TKE) data sets of ${ }^{252} \mathrm{Cf}(\mathrm{SF})$ (see Fig.1) exhibits different slopes as following: three data sets of Budtz-Jorgensen (open squares), Vorobyev (open diamonds) and Sing Shengyao (open down triangles) exhibit almost the same slope $\mathrm{dTKE} / \mathrm{d} v$ and a flat behaviour at low TKE values. Other three data sets of Bowman (open up triangles), Zeynalov (stars) and van Aarle (open circles) exhibit a lower slope $|\mathrm{dTKE} / \mathrm{d} v|$ than the three sets mentioned above and an almost linear behaviour over the entire TKE range. A flattening of $\langle v\rangle$ at low TKE is obtained by eliminating the wings of experimental $v(\mathrm{~A}, \mathrm{TKE})$ data of van Aarle. Details about these different behaviours can be found in [1]. The two experimental $<_{\varepsilon}>(\mathrm{TKE})$ data sets of ${ }^{252} \mathrm{Cf}(\mathrm{SF})$ ) exhibit 
different dTKE/d $\varepsilon$ slopes, too, (see Fig.2 and details in Ref.[1]). Different slopes are observed also in the case of experimental $<v>\left(\right.$ TKE) data of ${ }^{235} \mathrm{U}\left(\mathrm{n}_{\mathrm{th}}, \mathrm{f}\right)$ (see the upper part of Fig.3 and details in [1]).

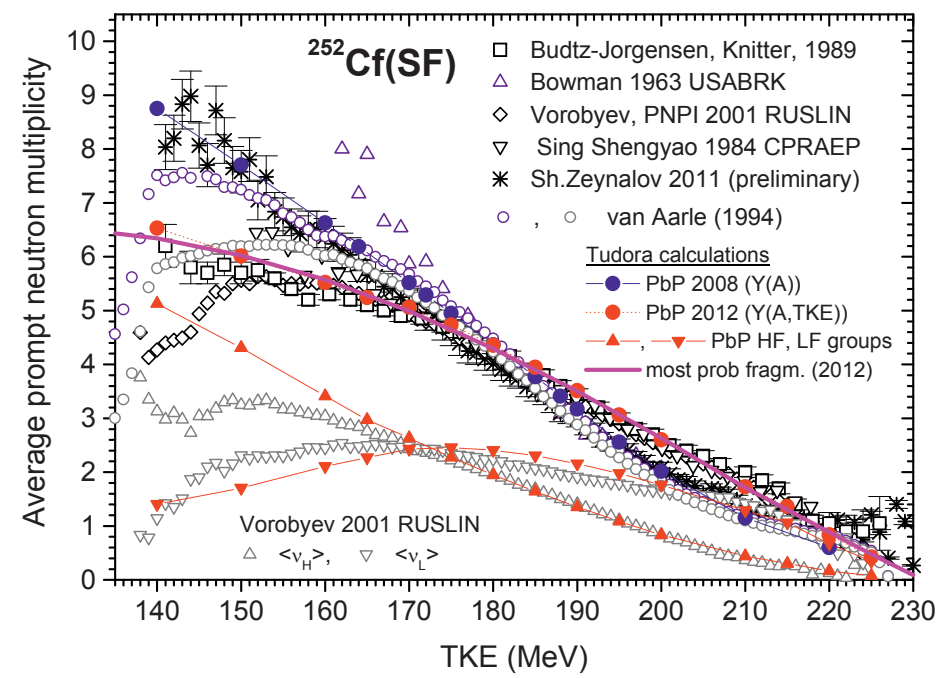

Fig.1: $\left\langle v>\right.$ (TKE) of ${ }^{252} \mathrm{Cf}(\mathrm{SF})$ : experimental data of Budtz-Jorgensen (open squares), Vorobyev (open diamonds), Sing Shengyao (open down triangles), Bowman (open up triangles), Zeynalov (stars), van Aarle (open circles). PbP results obtained by averaging $v(\mathrm{~A}, \mathrm{TKE})$ over $\mathrm{Y}(\mathrm{A})$ with full blue circles and over $\mathrm{Y}(\mathrm{A}, \mathrm{TKE})$ with full red circles. $\mathrm{PbP}$ results of $\left\langle v_{\mathrm{H}}>\right.$ and $\left\langle v_{\mathrm{L}}>\right.$ with full red up and down triangles in comparison with Vorobyev data (open grey up and down triangles). Most probable fragmentation result with bulk solid magenta line. Other lines connecting the calculation points are only to guide the eye.

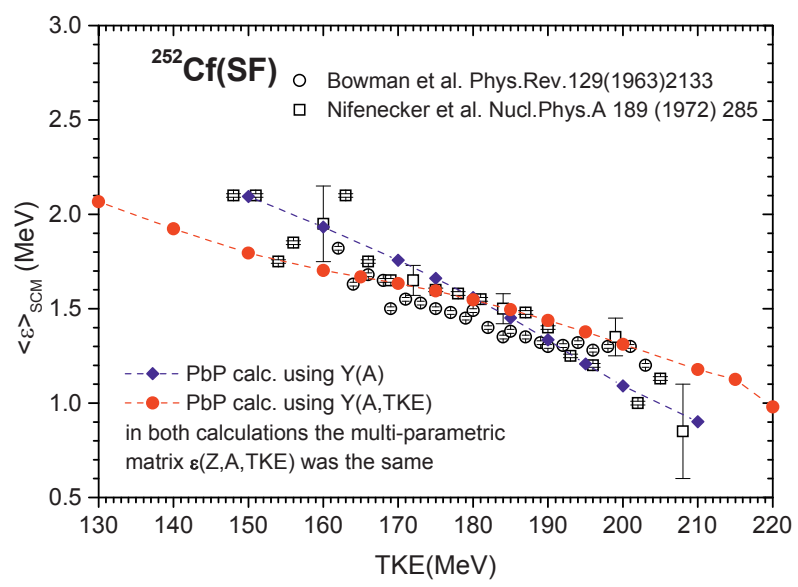

Fig.2: $<\varepsilon>$ (TKE) of ${ }^{252} \mathrm{Cf}(\mathrm{SF})$ : experimental data with open symbols, $\mathrm{PbP}$ results by averaging $\varepsilon(\mathrm{A}, \mathrm{TKE})$ over Y(A) (full blue diamonds) and over Y(A,TKE) (full red circles). Dashed lines are only to guide the eye.

The $<v>$ (TKE) result reported in [2], plotted with full blue triangles in Fig.1, is obtained by averaging the multi-parametric matrix $v(\mathrm{~A}, \mathrm{TKE})$ provided by the Point by Point $(\mathrm{PbP})$ model [2], over the experimental fission yield $\mathrm{Y}(\mathrm{A})$ of [3] (assumed independent on TKE). This result agrees well with the three experimental data sets exhibiting a lower slope $|\mathrm{dTKE} / \mathrm{d} v|$ (Bowman, Zeynalov and van Aarle). The $\langle v>$ (TKE) result plotted with full red circles describes very well the other three experimental data sets (Budtz-Jorgensen, Vorobyev and Sing Shengyao) exhibiting a higher $|\mathrm{dTKE} / \mathrm{d} v|$ slope and a flattening of $\langle v\rangle$ at low TKE values. This result was obtained by averaging the same matrix $v(\mathrm{~A}, \mathrm{TKE})$ of [2] over the double distribution Y(A,TKE) reconstructed from the 
experimental single distributions $\mathrm{Y}(\mathrm{A}), \mathrm{TKE}(\mathrm{A})$ and $\sigma_{\mathrm{TKE}}(\mathrm{A})$ reported in [3]. The $\mathrm{PbP}$ result of average multiplicities of the heavy and light groups $\left\langle v_{\mathrm{H}}\right\rangle,\left\langle v_{\mathrm{L}}\right\rangle$ plotted with full red up and down triangles in Fig.1, is in good agreement with the experimental data of Vorobyev (open grey up and down triangles). The $<\varepsilon>$ (TKE) results obtained by averaging the same $\mathrm{PbP}$ matrix $\varepsilon(\mathrm{A}, \mathrm{TKE})$ over Y(A) (full blue diamonds in Fig.2) and over the reconstructed double distribution Y(A,TKE) (full red circles in Fig.2) also exhibit different dTKE/d $\varepsilon$ slopes and are in agreement with the spread experimental data (open symbols). An interesting detailed analyse regarding the different slopes obtained by averaging $v(\mathrm{~A}, \mathrm{TKE})$ over a double distribution $\mathrm{Y}(\mathrm{A}, \mathrm{TKE})$ and the approximation by assuming an independent fission yield $\mathrm{Y}(\mathrm{A})$ is made in Ref.[4] for the case of ${ }^{252} \mathrm{Cf}(\mathrm{SF})$. The $\mathrm{PbP}$ results of $\langle v\rangle$ (TKE) previously obtained [2] by averaging over a single distribution Y(A) (assumed independent on TKE) and presently by averaging over a double distribution Y(A,TKE) (see Ref.[1]) are in agreement with the Monte-Carlo calculations reported in the analyse of Ref.[4]

In the case of ${ }^{235} \mathrm{U}\left(\mathrm{n}_{\mathrm{th}}, \mathrm{f}\right)$ similar results consisting in a higher slope $|\mathrm{dTKE} / \mathrm{d} v|$ and a flattening of $\langle v\rangle$ at low TKE were obtained by averaging over Y(A,TKE) (full red circles in the upper part of Fig.3) compared to the previous result of Ref.[2] when the approximation by averaging over Y(A) was used (blue circles in the upper part of Fig.3).

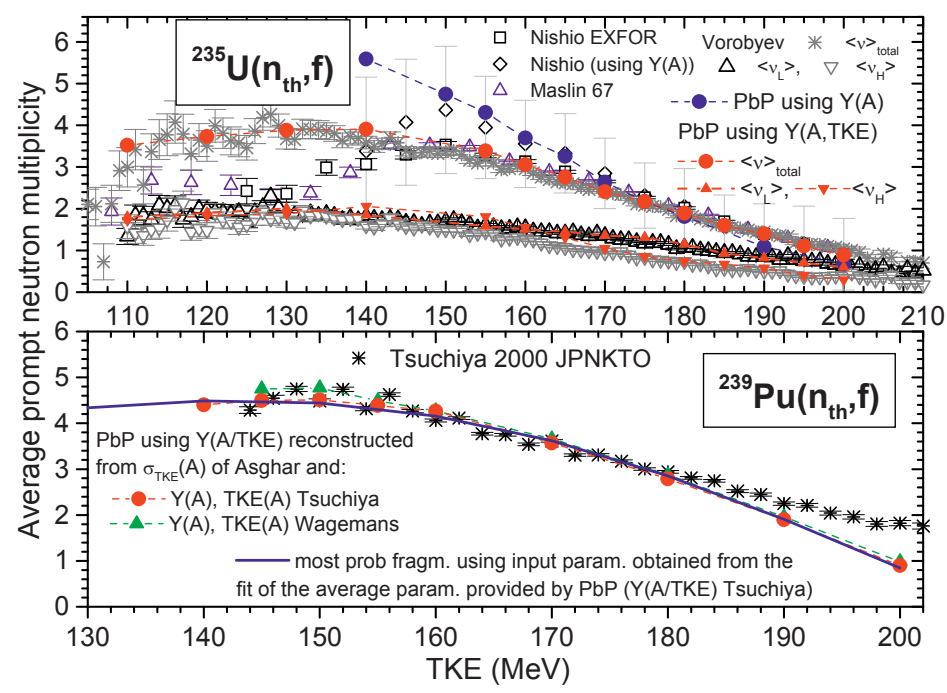

Fig.3: Upper part $<v>(T K E)$ of ${ }^{235} \mathrm{U}\left(\mathrm{n}_{\mathrm{th}}, \mathrm{f}\right)$ : $\mathrm{PbP}$ results obtained by averaging over $\mathrm{Y}(\mathrm{A})$ (full blue circles) and over $\mathrm{Y}(\mathrm{A}, \mathrm{TKE})$ (full red circles) in comparison with experimental data (different open symbols). PbP result of $\left\langle v_{\mathrm{L}}>\right.$ and $\left\langle v_{\mathrm{H}}>\right.$ with full red up and down triangles in comparison with experimental data (open up and down triangles). Lower part $\left\langle v>(\mathrm{TKE})\right.$ of ${ }^{239} \mathrm{Pu}\left(\mathrm{n}_{\mathrm{th}}, \mathrm{f}\right) \mathrm{PbP}$ results obtained by averaging over $\mathrm{Y}(\mathrm{A}, \mathrm{TKE})$ reconstructed from experimental single distributions of Tsuchiya (full circles) and Wagemans (full triangles) in comparison with experimental data (stars). Most probable fragmentation result is plotted with blue solid line.

As it can be seen $<v>$ (TKE) obtained by averaging over Y(A,TKE) (full red circles) as well as $\left\langle v_{\mathrm{L}}>\right.$ and $\left\langle v_{\mathrm{H}}\right\rangle$ (full red triangles) describe well the majority of experimental data sets.

In the case of ${ }^{239} \mathrm{Pu}\left(\mathrm{n}_{\mathrm{th}}, \mathrm{f}\right),($ lower part of Fig.3) the behaviour of the unique experimental data set (stars) suggests that only a $<v>$ (TKE) result obtained by averaging over a double distribution $\mathrm{Y}(\mathrm{A}, \mathrm{TKE})$ can describe these data. The $\langle v\rangle(\mathrm{TKE})$ results (full red circles and green triangles) obtained by averaging the $\mathrm{PbP}$ matrix $v(\mathrm{~A}, \mathrm{TKE})$ over two reconstructed $\mathrm{Y}(\mathrm{A}, \mathrm{TKE})$ distributions (from experimental single distributions of Tsuchiya and Wagemans) are rather close to each other and in agreement with the experimental data (for details see Ref.[1]).

The experimental fission fragment distributions of ${ }^{234} \mathrm{U}(\mathrm{n}, \mathrm{f})$ recently measured at IRMM at 14 incident energies (En) covering the range $0.3-5 \mathrm{MeV}$, allow to calculate for the first time prompt neutron emission quantities as a function of TKE at many En. Calculations of $\langle v>$ (TKE) at three En 
values are given as example in Fig.4. The $<v>$ (TKE) results at many En revealed two interesting facts: a) the slope dTKE/d $v$ does not vary with En and b) the flattening of $\langle v\rangle$ at low TKE values is more pronounced at low incident energies (see Fig.4).

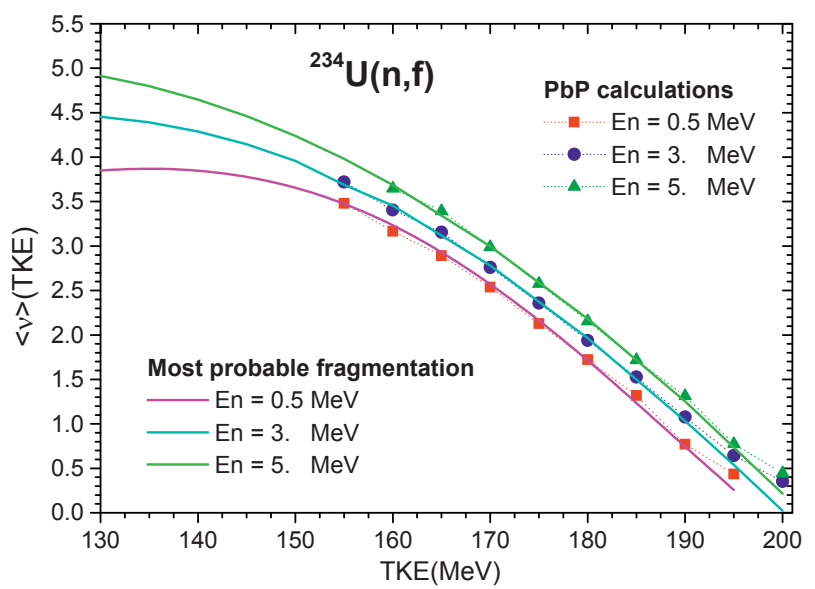

Fig.4: ${ }^{234} \mathrm{U}(\mathrm{n}, \mathrm{f})<v>(\mathrm{TKE})$ at three En values, $\mathrm{PbP}$ calculations (different full symbols) and most probable fragmentation results using the average model parameter dependences on TKE with solid lines.

\section{Average model parameter dependences on TKE}

The average values of model parameters: energy release $<$ Er $>$, average neutron separation energy from $\mathrm{FF}<\mathrm{Sn}>$ and level density parameter $<a>$ (traditionally given as $<\mathrm{C}>=\mathrm{A}_{0} /<a>$ with $\mathrm{A}_{0}$ the mass number of the fissioning nucleus) can be obtained at a given value of TKE by averaging the parameters of fragment pairs calculated at the respective TKE value over Y(A,TKE). For all studied fissioning systems these parameters exhibit nice and regular behaviours that can be fitted well. An example is given in Fig.5 (PbP results with black squares and appropriate fits with red lines). The obtained parameter dependences on TKE are used in the most probable fragmentation approach. The resulted $<v>$ (TKE) (plotted with bulk solid lines in Figs.1, 3 and 4) are very close to the PbP results and describe well the existing experimental data.

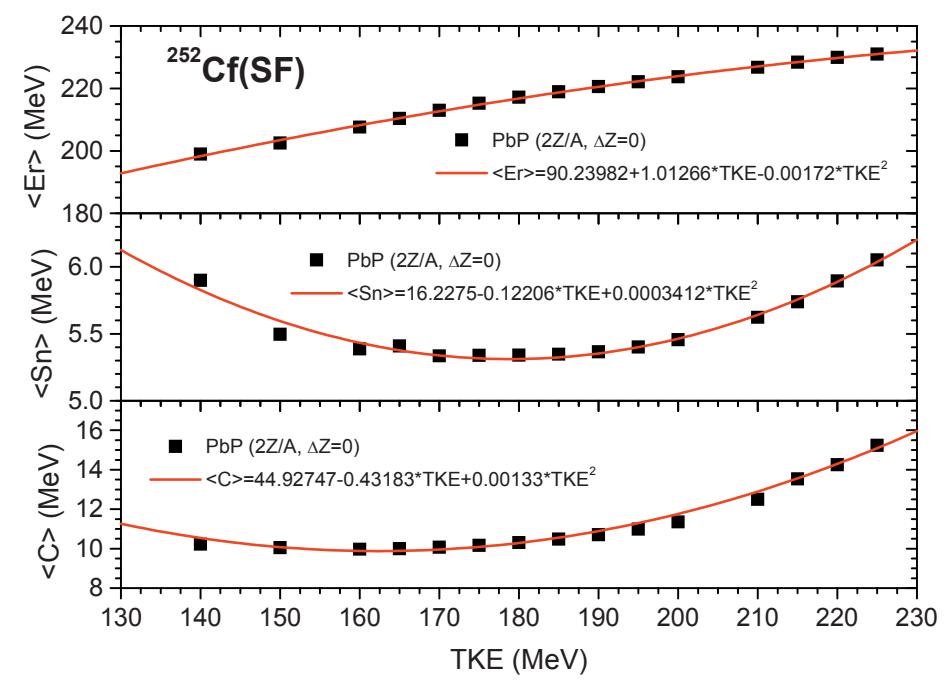

Fig.5 ${ }^{252} \mathrm{Cf}(\mathrm{SF})$ average model parameters as a function of TKE, obtained from the PbP treatment (full squares) and appropriate fits (solid lines). 
The most probable fragmentation approach with average model parameter dependences on TKE resulted from the $\mathrm{PbP}$ treatment has as advantages to provide results at many TKE values in a very short computing time compared with other models (like the PbP model and the Monte-Carlo treatment) and to give results also outside the TKE range where the $\mathrm{PbP}$ model is applicable.

\section{Consistency of PbP model calculations}

The consistency of present calculations can be proved by the following verification. The total average values of model parameters $(<\mathrm{Er}>,\langle\mathrm{TKE}>,<\mathrm{Sn}>,<\mathrm{C}>$ ) for a given fissioning system at a given excitation energy are usually obtained by averaging the parameters given as a function of fragment mass number ( $\operatorname{Er}(\mathrm{A}), \operatorname{TKE}(\mathrm{A}),<\mathrm{Sn}>(\mathrm{A}),<\mathrm{a}>(\mathrm{A})$ ) over the fragment mass distribution $\mathrm{Y}(\mathrm{A})$ [5]. On the other hand total average parameters can be obtained by averaging the parameters as a function of TKE $(<\mathrm{Er}>(\mathrm{TKE}),<\mathrm{Sn}>(\mathrm{TKE}),<\mathrm{a}>$ (TKE)) over the TKE distribution Y(TKE). For all studied fissioning systems the deviation of parameters obtained by averaging over Y(TKE) from the values obtained by averaging over $\mathrm{Y}(\mathrm{A})$ is less than $2 \%$. Also the total average prompt neutron multiplicity $\left\langle v_{\mathrm{p}}\right\rangle$ can be obtained by averaging $v(\mathrm{~A})$ over $\mathrm{Y}(\mathrm{A})$ and by averaging $\langle v\rangle$ (TKE) over $\mathrm{Y}(\mathrm{TKE})$. The deviation of $\left\langle v_{\mathrm{p}}\right\rangle$ obtained by averaging over $\mathrm{Y}$ (TKE) from the value obtained by averaging over $\mathrm{Y}(\mathrm{A})$ does not exceed 3-4\% (see Ref.[1] for details).

\section{Conclusions}

The slope $d T K E / d v$ and the flattening of $\langle v\rangle$ at low TKE values, exhibited by a great part of experimental $\langle v>$ (TKE) data sets, are well described when the multi-parametric matrix $v(\mathrm{~A}, \mathrm{TKE})$ provided by the $\mathrm{PbP}$ model is averaged over a double distribution $\mathrm{Y}(\mathrm{A}, \mathrm{TKE})$. The approximation of averaging the same matrix $v(\mathrm{~A}, \mathrm{TKE})$ over an yield $\mathrm{Y}(\mathrm{A})$ assumed independent on TKE, leads to $<v>$ (TKE) with lower slopes $\mathrm{dTKE} / \mathrm{d} v$ and an almost linear behaviour over the entire TKE range, in agreement with another part of experimental data sets.

These facts are supported by two different treatments: the PbP model (as described in Ref.[1] and references therein) and the Monte-Carlo calculations (as described in Ref.[4] and references therein) leading in both cases - by averaging over a double distribution $\mathrm{Y}(\mathrm{A}, \mathrm{TKE})$ and over a single one $\mathrm{Y}(\mathrm{A})-$ to similar results of $<v>$ (TKE).

In the case of ${ }^{234} \mathrm{U}(\mathrm{n}, \mathrm{f})$ the $\mathrm{PbP}$ calculation of $\langle v>$ (TKE) at many incident energies using the recent experimental Y(A,TKE) distributions measured at IRMM, revealed that the slope dTKE/dv does not vary with the incident energy and the flattening of $\langle v\rangle$ at low TKE values is more pronounced at low incident energies.

The average values of model parameters as a function of TKE obtained from the PbP treatment exhibit nice and regular behaviours that can be fitted well. The resulted parameter dependences on TKE allow the use of the "most probable fragmentation approach" (Los Alamos model with subsequent improvements) having as advantages to provide results at many TKE values in a very short computing time compared to the Point by Point and Monte Carlo treatments.

\section{References}

1. A. Tudora, Ann. Nucl. Energy 2012 in press, http://dx.doi.org/10.1016/j.anucene.2012.10.007

2. A. Tudora, Ann. Nucl. Energy 35 (2008) 1-10

3. F.-J. Hambsch, S. Oberstedt, Nucl. Phys.A 617 (1997) 345-355

4. D. Regnier, O.Litaize, O. Serot, Nucl. Sci. Eng. 2012 in press.

5. A. Tudora, Ann. Nucl. Energy 36 (2009) 72-84 